\title{
Pandemics and Mental Health: an Unfortunate Alliance
}

\author{
Shinu Kuriakose ${ }^{1}$ \\ Accepted: 28 August 2020 / Published online: 11 September 2020 \\ (C) Springer Nature Switzerland AG 2020
}

\begin{abstract}
Mental health issues not only affect the person but society as a whole. This is especially apparent during times of pandemics or other social unrest situations as currently seen during the COVID-19 crisis. It can manifest itself as violence (towards or from the mentally ill person), increased substance use, increased overcrowding of our prisons, tackling with comorbid medical conditions which have worsened due to lack of initial attention (a particularly big problem among the mentally ill), increased strain on tax payers, and overall affecting the quality of everyone's life. Furthermore, mental health maladies can cause increase work absenteeism and poor work performance and decreased economic productivity. The stigma associated with mental health also leads to poor funding form policy makers, as there is a lack of forceful advocacy in dealing with these issues. The World Health Organization (WHO) estimates that the loss of productivity due to mental health disability accounts to close to $5 \%$ of the gross national product of the European Union (WHO 2019). One of the most crucial causes of lack of mental screening, recognition, and treatment is the limited availability of mental health trained providers. The physician assistant profession is in a unique role to fill this niche as they have been trained as generalists who specialize only when getting trained in their specific field during their employment. Additionally, the fact that PAs do have lateral mobility does lend this profession to meet the needs of society, especially in the psychiatric fields, in a prompt and competent manner. The need for physician assistants (PAs) in psychiatry and addiction medicine is a nationwide trend resulting in existing and emerging shortages of psychiatric and addiction medicine providers. They can provide relatively inexpensive, easily accessible, and good quality care to their clients.
\end{abstract}

Keywords Psychiatry $\cdot$ Mental health $\cdot$ Physician assistant $\cdot$ Access to care $\cdot$ Disability

\section{Proposal for a Physician Assistant Behavioral Medicine Fellowship}

Provide graduate physician assistants with the knowledge, skills, and training to manage the care of patients in the areas of psychiatry and addiction medicine in varied settings in a timely and competent manner.

Proposal for a competent, efficient, cost-effective, timely, and skilled provider pool to be trained in a professional manner for treatment of psychiatric condtions, reducing stigma and ongoing education on a very misunderstood topic

This article is part of the Topical Collection on Medicine

Shinu Kuriakose

skuria06@nyit.edu

1 School of Health Professions, New York Institute of Technology, Old Westbury, NY, USA

\section{Disparities in Mental Health in the USA}

Mental illness remains one of the leading causes of disability in the USA, reinforcing the need for early detection, timely behavioral and psychopharmacological therapy, and consistent follow-up [1]. According to the National Institute of Mental Health (NIMH), 57.7 million people over the age of 18 (26.2\% of the population) suffer from a diagnosable mental health disorder in any given year. Mental health medications were second among all the classes of medications dispensed in the USA with an expenditure of 25.5 billion dollars [13]. The atypical antipsychotic medication, aripripazole, was the second most dispensed medication with spending of 5.9 billion dollars [13]. In the last decade, mental health medication utilization has seen the largest volume growth [10]. Recently, mental illness has made quite a few headlines for the wrong reasons. Violent acts have been prominently been performed by folks with mental health issues over the last 20 years due to the individual's psychiatric decompensation and unable to be 
treated in a timely manner by a competent clinician. It has been revealed quite often in these cases that family members and friends of these individuals recognized the mental malady in these patients but were helpless to refer them to resources due to financial barriers and lack of access to care. One common thread in all these shootings was noncompliance with psychiatric medications: a condition often seen due to a lack of affordable mental health providers.

This mental health pandemic crisis is further exacerbated due to the ready availability of weapons, potent drugs (prescribed or illicit), and other mechanisms which have led to violent outcomes. Patients suffering from psychiatric conditions are often evaluated by their primary care providers (if available) or a harried emergency room provider and given "band-aid" service which does not involve adequate follow through in terms of medication compliance and further treatment. It remains an unfortunate fact that most patients receiving psychopharmacological modalities are in the US prison system: a clear example of patients being detained against their will due to an outcome of their mental disability [6]. The vast majority of clinical providers, untrained in psychiatry, although trying their best to treat their patients in the most meaningful way possible are not credentialed or experience in the robust treatment of mental health patients: a disservice to their well-intentioned treatment plans and to the patients.

As a practicing psychiatric physician assistant for the last 19 years, noncompliance with psychiatric medications leading to inpatient psychiatric hospitalizations is seen in most behavioral medicine patients. This is compounded by the fact that patients often self-medicate themselves (in a bid to be symptom free) with illicit street drugs and alcohol: a morbid cocktail which leads to further psychiatric decompensation [7]. Often the illicit substances obtained from street-level drug dealers counteract not only the prescribed medications patients are supposed to be on but also lead to multiple medical disabilities, further compounding the health and well-being of these individuals. Studies had concluded that multiple psychiatric admissions often lead to lower mental health baseline when compared with previous psych admissions [11]. The mitigation of mental health disabilities to include psychiatric conditions as meeting the criteria in the Diagnostic and Statistical Manual of Psychiatry (DSM-5), substance abuse, interpersonal and stranger violence, and poor comorbid medical outcomes can be overcome with availability and accessibility of providers. The physician assistant (PA) provider can play a robust and crucial part in reducing wait times to see a competent clinician, prescribe psychotropic medications as appropriate, engage in psychological and behavior counseling, and refer patients to available resources. PAs, as masterlevel clinicians, already graduate with a fundamental knowledge of medicine, and this coupled with advanced psychiatric practical and theoretical training in an organized format can arise and meet this need. There continues to be increased recognition of this fact, but legislative and political barriers still need to be overcome to clear the hurdles so PAs can meet their patients at the point of their need [15].

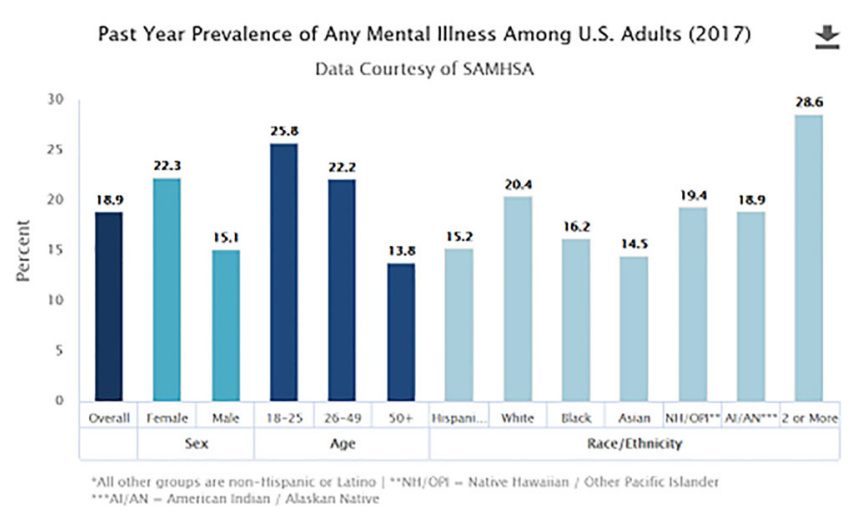

The Surgeon General's report on mental health "documented the existence of mental health disparities, noting that while mental illness is at least as prevalent among racial and ethnic minorities as in whites, minorities have less access to and are less likely to use mental health services, and receive poorer quality mental health care. Consequently, minority communities have a higher proportion of individuals with unmet mental health needs" (NIH.gov, 2018). The barriers to receiving affordable and comprehensive mental health are greatly exacerbated in the context of social or natural disasters. One of the examples cited by the National Institute of Health is New Orleans post Katrina: a true disaster in terms of mental health disparities. Multiple inpatient psychiatric units and outpatient crisis units were destroyed in the wake of Hurricane Katrina and not even one has reopened. The few remaining mental health clinics report that $50-60 \%$ of adults and $20 \%$ of children suffer from some diagnosable mental health condition (depression, posttraumatic stress disorder, anxiety, substance dependence), and this fact is exacerbated by not having enough mental health clinicians [8]. Per the NIH, mental health is the most significant healthcare challenge facing the New Orleans health system. College students are a special population where the rates of mental health conditions have increased substantially due to the increased pressures of social media, keeping up with their peers, growing up in an environment with exposure to increased domestic and stranger violence, and lack of adequate support systems. There need to be providers based in student health services who need to be adequately trained in dealing with college students presenting with such issues. Most of these student health services are staffed with advanced practice non-physicians (physician assistants and nurse practitioners). Therefore, PAs in these situations, if trained to screen, diagnose, counsel appropriately, and refer when needed to outside resources, can be a lifeline to these students who suffer from increased stress in the context of their college experience. Suicide continues to be one of the major 
reasons for death in this population in conjunction with drug overdoses. Students who are not appropriately treated at this time will also suffer the consequences of bad academic outcomes coupled with increased interpersonal conflicts with family and friends [9]. Therefore, early intervention by trained providers can provide a much-needed lifeline to this population.

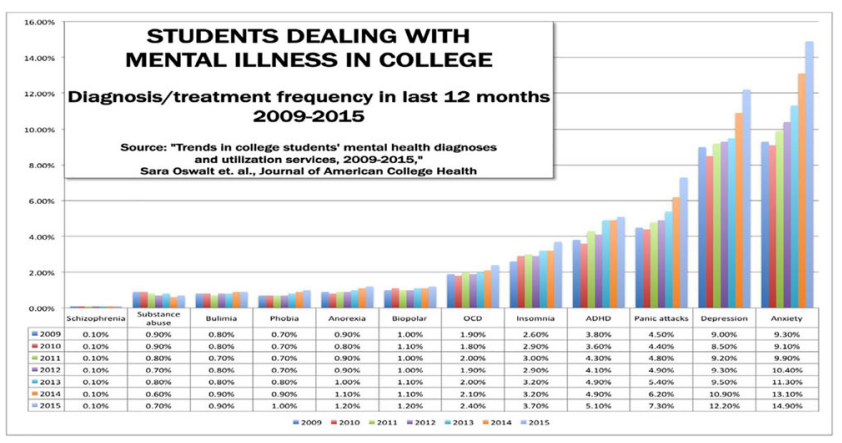

It remains a fact that individuals residing in lower socioeconomic neighborhoods, people of color, families struggling economically, and children home alone most of the day due to their parent's forced to work to support them with multiple jobs have the worst outcomes in their mental health conditions. This is further worsened as the accessibility of mental health caregivers in these communities remains low as providers are in a position to choose practice locations in which patients can give them their fees without depending on private or governmental insurance plans. These private pay clinicians often are paid 2-3 times more than a clinician staffing a clinic economically challenged neighborhood.

According to the Health Resources and Services Administration (2010), as of March 2010, there are severe shortage of health clinicians across the nation with 3431 designated mental Health Professions Shortage Areas (HPSAs) in the nation; additionally, 5553 prescribing mental health clinicians would be needed to meet this shortage [3].

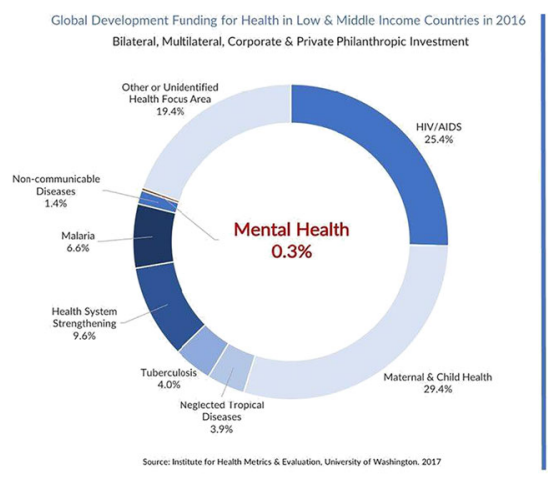

DEPRESSION IS THE LEADING CAUSE OF DISABILITY WORLDWIDE

MENTALHEALTH (INCLUDING DEPRESSION) RECEIVED $\$ 128 \mathrm{M}$ OF THE

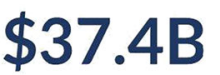

TOTALFUNDING
The United States Census Bureau estimates that the US population is going to increase by 50 million by 2025 but the number of physicians is only expected to increase by 54,000 with worst shortage in specialty areas, like psychiatry [5]. It has been recognized that the United States is suffering from a severe dearth of prescribing mental health clinicians (psychiatrist, nurse practitioner, physician assistants) at a time when patients are increasingly seeking this care. Ninety-six percent of all counties in the United States has a severe mental health clinician shortage [12]. According to the Health Resources and Services Administration (2015), as of March 2010, there are severe shortage of health clinicians across the nation with 3,431 designated mental-health Health Professions Shortage Areas (HPSAs) in the nation; additionally, 5,553 prescribing mental-health clinicians would be needed to meet this shortage [4].

Another barrier faced by mental health clients is the lack of funding provided for increased mental health and addiction coverage. The National Alliance of Mental Health (NAMI) states that 1.6 billion dollars were cut from non-medicaid state mental health budgets.

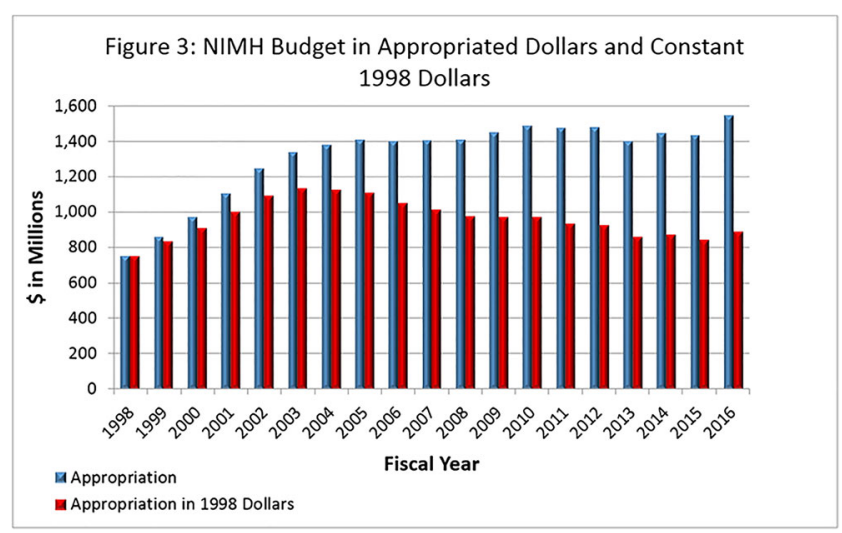

National Institute of Mental Health (NIMH): Mental Health Appropriated funds decrease between 1998-2016

This is at the time when mental health consumers are increasingly being forced to pay higher out-of-pocket costs. This particularly hits this population hard as often they are unemployed and rely on public subsidies. This scenario is particularly true in times of economic stress caused by pandemics or other disruption in the social fabric scenario where individuals with low wage salaries are often the ones to get their hours cut, lose their jobs, or have no other mechanisms to work from other settings. 
Figure 2. Out-of-Pocket Costs for Mental Health Services among Adults Aged 18 or Older Who Received Outpatient:- or Inpatient:- Mental Health Services in the Past Year: 2005 to 2009

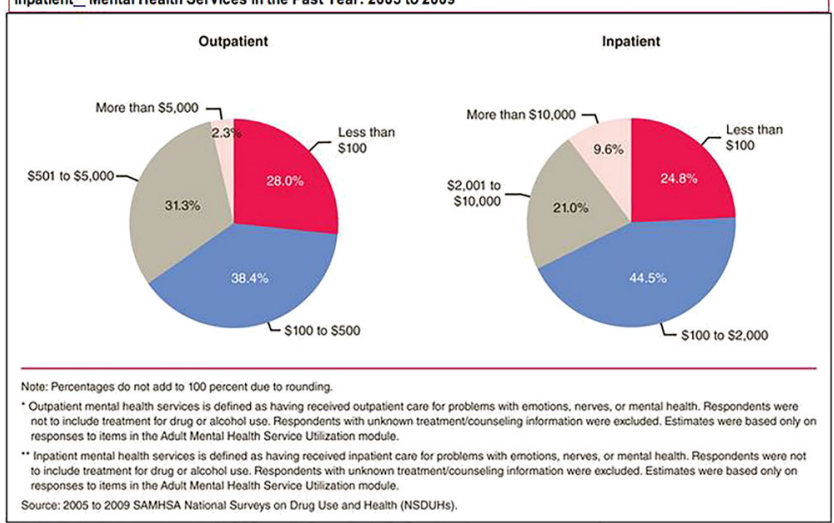

Mental health issues not only affect the person but society as a whole. It can manifest itself as violence (towards or from the mentally ill person), increased substance use, increased overcrowding of our prisons, tackling with comorbid medical conditions which have worsened due to lack of initial attention (a particularly big problem among the mentally ill), increased strain on tax payers, and overall affecting the quality of everyone's life. The stigma associated with mental health also leads to poor funding form policy makers, as there is a lack of forceful advocacy in dealing with these issues. There needs to be increased recognition that mental health illness is not due to a weak character of an individual or lack of will power; rather it needs to be viewed as a medical condition for which treatment is available and the prognosis is good if timely, adequate, and competent treatment is received. The World Health Organization (WHO) estimates that loss of productivity due to mental health disability accounts to close to $4 \%$ of the gross national product of the European Union [14].

The need for physician assistants (PAs) in psychiatry and addiction medicine is a nationwide trend resulting in existing and emerging shortages of psychiatric and addiction medicine providers. They can provide relatively inexpensive, easily accessible, and good quality care to their clients. PAs, prior to applying to physician assistant programs which typically are between 24 and 36 months in length, already have a bachelor's degree from an accredited college or university. Attaining the PA credential typically bestows the PA with a master's degree, and there are PAs who might opt for further fellowship training in fields such as emergency medicine, orthopedics, critical care, surgery, etc. Psychiatry is one of the fields, where there is a need for expanded and robust fellowship training for PAs due to the support they can provide to the medical establishment and the competent care whey patients can receive. It is a win-win situation for all stakeholders in this battle.
There could be many different types of scenarios which could form a framework for licensed PAs to undergo further training, in a fellowship format, to include both practical clinical training and classroom didactic theory under experts in this field which could lead them to pass an assessment protocol and embark on a career treating and caring for people who have historically been shunned by the medical establishment. Decreasing stigma, increasing education for the population as a whole, for families of patients who suffer from these conditions, and imbibing hope in patients themselves will go a long way to help society as a whole to appreciate and respect these individuals who are suffering [2]. It will also take advocacy by providers and other stakeholder in this field to encourage lawmaker to work with governmental agencies and private insurance companies to ensure reimbursements are fair and resources are available for these afflicted patients.

Proposed sample program description:

- Full-time 12-month program.

- Proposed start date January 2, 2021.

- 2-4 physician assistants accepted annually (these are licensed physician assistants who are already practicing in various medical fields including psychiatry).

- 30 credit curricula including a combination of didactic courses and clinical clerkships.

- Master of Science degree/certificate with a concentration in psychiatry or addiction medicine.

- Stipend provided by medical institution yearly with benefits. This expense may be offset by the billing done by the PA's for their services.

- 2-year employment commitment with medical institution (at the discretion of the Department of Psychiatry).

- Commitment by applicants to serve in an underserved, low-income neighborhood after graduation.

Overall, it is going to take a joint effort by various health agencies, educational institutions, policy makers, advocacy groups for the mentally ill, and medical societies to try to grapple with this immense problem and find a sustainable and effective solution, one which might be expensive and arduous in the short term but with great potential and cost saving in the long term. These patients deserve better and it behooves us as a society to ensure that nobody is left behind.

\section{Compliance with Ethical Standards}

Conflict of Interest The author declares that he/she has no conflict of interest. 
Human and Animal Rights and Informed Consent The manuscript in part or in full has not been submitted or published anywhere. This study did not involve any human subjects or animals.

\section{References}

1. Filipčić IŠ, Bajić Ž, Filipčić I. The onset and accumulation of physical multimorbidity in severe and common mental disorders. Curr Opin Psychiatry. 2020. https://doi.org/10.1097/yco. 0000000000000635.

2. Guerra SG, Berbiche D, Vasiliadis H. Changes in instrumental activities of daily living functioning associated with concurrent common mental disorders and physical multimorbidity in older adults. Disabil Rehabil. 2020:1-9. https://doi.org/10.1080/ 09638288.2020 .1745303$.

3. Health Resources and Services Administration. 2010. Retrieved from https://bhw.hrsa.gov/shortage-designation/hpsas. Accessed 9/2/2020.

4. Health Resources and Services Administration. 2015. Retrieved from https://www.hrsa.gov/behavioral-health. Accessed 9/2/2020

5. Health Resources and Services Administration (HRSA). Shortage designation: HPSAs, MUAs \& MUPs. 2018. Retrieved from http:// bhpr.hrsa.gov/shortage.

6. National Institute of Mental Health. 2015. Retrieved from http:// www.nimh.nih.gov/health/publications/the-numbers-countmental-disorders-in-america/index.shtml. Accessed 3/15/2015.

7. National Institute of Mental Health. 2017. Retrieved from http:// www.nimhd.nih.gov/hdFactSheet.asp?print=1. Accessed 8/17/ 2017.

8. National Institute of Mental Health. 2018. Retrieved from https:// www.nimh.nih.gov/health/statistics/mental-illness.shtml. Accessed 9/2/2020.

9. Niederkrotenthaler T, Mittendorfer-Rutz E, Thurner S, Endel G, Klimek P. Healthcare utilization, psychiatric medication and risk of rehospitalization in suicide-attempting patients with common mental disorders. Aust N Z J Psychiatry. 2019;54(4):409-22. https://doi.org/10.1177/0004867419895112. Accessed 7/25/2019.
10. Pharmacy Times. 2013. Retrieved from http://www. pharmacytimes.com/publications/issue/2013/July2013/Top-200Drugs-of-2012.

11. Šprah L, Dernovšek MZ, Wahlbeck K, Haaramo P. Psychiatric readmissions and their association with physical comorbidity: a systematic literature review. BMC Psychiatry. 2017;17:2. https:// doi.org/10.1186/s12888-016-1172-3. Accessed 2/21/2017.

12. Thomas, et al. County level estimates of mental health professional shortage in the United States. Psychiatr Serv. 2009;60:1323-228. https://doi.org/10.1176/appi.ps.60.10.1323.

13. Vijay A, Becker JE, Ross JS Patterns and predictors of off-label prescription of psychiatric drugs. PLOS ONE 2018;13(7): e0198363. https://doi.org/10.1371/journal.pone.0198363.

14. World health Organization. 2013. Retrieved from https://www. who.int $/$ mental health/in the workplace/en/\#: :text=A\% 20 recent $\% 20 \mathrm{WHO} \% 2 \mathrm{Dled} \% 20$ study,or\%20getting\%20work\% 20 is\%20protective. Accessed May 2019.

15. World health Organization. 2019. Retrieved from http://www.euro. who.int/_data/assets/pdf_file/0008/134999/e94837.pdf. Accessed $3 / 4 / 2019$

\section{Further Reading}

16. Buhagiar K, Templeton G, Osborn DP. Recent physical conditions and health service utilization in people with common mental disorders and severe mental illness in England: comparative crosssectional data from a nationally representative sample. Eur Psychiatry. 2020;63(1):e19. https://doi.org/10.1192/j.eurpsy.2020. 22.

17. Daly R. AAMC issues alert about looming physician shortage. Psychiatr News. 2009;44(2):4 Retrieved from http://pn. psychiatryonline.org/content/44/2/4.2.full.

Publisher's Note Springer Nature remains neutral with regard to jurisdictional claims in published maps and institutional affiliations. 